See Article page e389.

\section{Commentary: Unraveling the mystery of transient innate capacity for neonatal heart regeneration following injury}

\author{
Devendra K. Agrawal, MSc, PhD (Biochem), PhD \\ (Med Sci), MBA, MS(ITM), FAAAAI, FAHA, FAPS, \\ FIACS
}

Survival of the fetal heart in the hypoxic intrauterine environment depends on the nutrition, respiration, and waste elimination provided by the placenta. However, such dependency is lost at birth. In the extrauterine environment, the neonatal heart functions on its own due to wellorchestrated and rapid adaptations in various anatomical, cellular, molecular, and physiologic processes. ${ }^{1}$ In addition, several epigenetic factors and innate immune response trigger a switch in cardiomyocytes from hyperplastic mode to hypertrophy with limited renewal capacity when encountering with growth stimuli. Any insult or injury may perturb the adaptive process, leading to pathophysiologic changes. However, specific fetal genes in the neonatal cardiomyocytes are upregulated following injury to promote regenerative capacity of the heart, which could most likely be due to the proliferation of pre-existing cardiomyocytes and possibly the differentiation of embryonic stem cells into cardiomyocytes that undergo a switch from glycolytic to oxidative phase in energy substrate metabolism,

From the Department of Translational Research, Western University of Health Sciences, Pomona, Calif.

The research work of Dr Agrawal is supported by research grants R01 HL144125 and R01HL147662 from the National Institutes of Health. The content of this article is solely the responsibility of the authors and does not necessarily represent the official views of the National Institutes of Health.

Disclosures: The author reported no conflicts of interest.

The Journal policy requires editors and reviewers to disclose conflicts of interest and to decline handling or reviewing manuscripts for which they may have a conflict of interest. The editors and reviewers of this article have no conflicts of interest.

Received for publication Aug 30, 2021; revisions received Aug 30, 2021; accepted for publication Aug 31, 2021; available ahead of print Sept 4, 2021.

Address for reprints: Devendra K. Agrawal, MSc, PhD (Biochem), PhD (Med Sci), MBA, MS(ITM), FAAAAI, FAHA, FAPS, FIACS, Department of Translational Research, Western University of Health Sciences, 309 E Second St, Pomona, CA 91766-1854 (E-mail: DAgrawal@WesternU.edu).

J Thorac Cardiovasc Surg 2022; 164:e407-8

$0022-5223 / \$ 36.00$

Copyright (c) 2021 by The American Association for Thoracic Surgery

https://doi.org/10.1016/j.jtcvs.2021.08.069
Q Check for updates

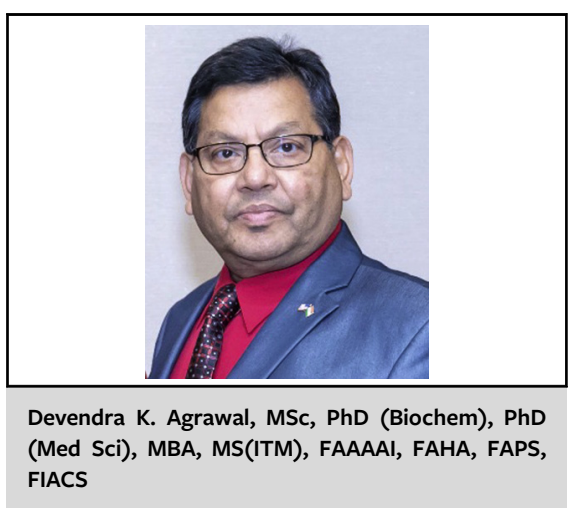

CENTRAL MESSAGE

The neonatal heart regeneration model following injury is a promising tool to unravel the complexity of heart regeneration.

driving the differentiation of embryonic stem cells into mature cardiomyocytes. ${ }^{2}$

The regenerative capability of the injured heart following myocardial infarction has been observed in mice, rats, and piglet models and in a newborn human case. ${ }^{3-8}$ The article published in this issue of the Journal by Wang and colleagues ${ }^{9}$ reports the age-dependent transient cardiac regenerative phenotype after myocardial infarction in the neonatal leporine model. The authors report a significant reduction in ejection fraction after postnatal day 1 myocardial infarction compared with sham, but the ejection fraction was same at 3 weeks. However, the decrease in ejection fraction did not return to the level of sham control if the coronary ligation was performed on day 4 or day 7 . The regeneration in the injured area after postnatal day 1 myocardial infarction is most likely due to the migration and proliferation of existing cardiomyocytes from the peri-infarct area. The transient capability of natural heart regeneration was lost within a week. This is perhaps the first study following ischemic injury in the newborn white rabbits. More importantly, the findings support the concept of conserved intrinsic mechanisms of neonatal cardiac regeneration in mammalian cardiomyocytes after myocardial infarction involving the re-expression of fetal genes and transcriptional programs that control cell proliferation and regenerative process (Figure 1). The underlying molecular mechanisms of the loss of regenerative capacity of the heart remain unclear. 

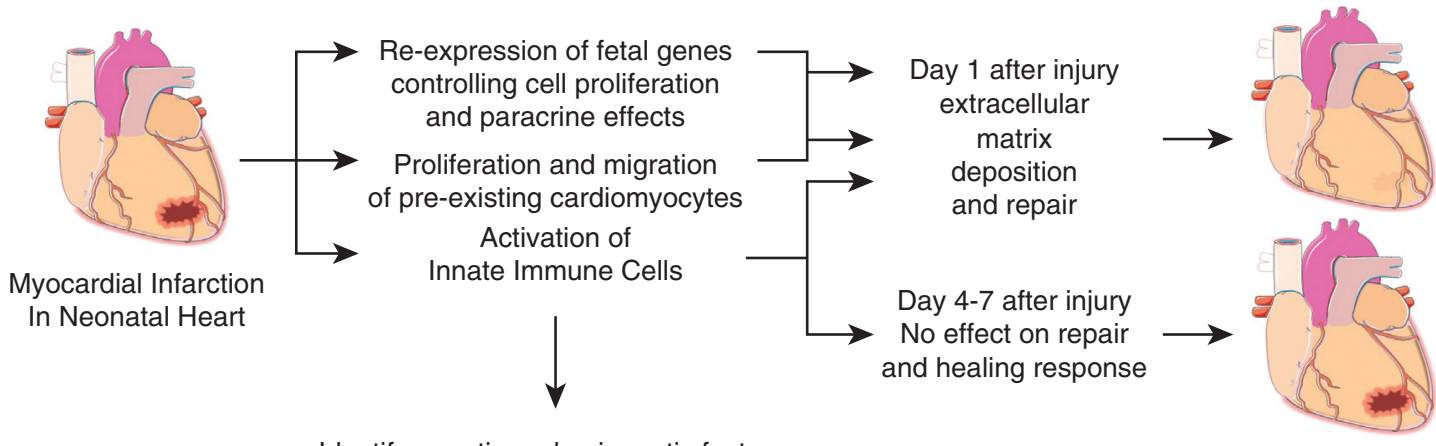

Identify genetic and epigenetic factors, fetal genes and innate immune response in neonatal heart regeneration model

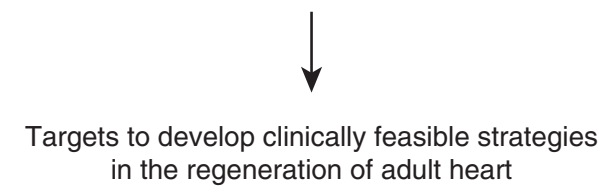

FIGURE 1. Schematic diagram showing the repair and healing of the neonatal heart 1 day after myocardial infarction, whereas on days $4-7$, there was no repair and healing. Further knowledge from such neonatal heart regeneration models would help in developing strategies to reprogram adult cardiomyocytes and induce immunomodulation to effectively repair and regenerate adult heart following myocardial infarction and other injuries.

The neonatal mammalian model of myocardial regeneration following injury, as reported by Wang and colleagues, ${ }^{9}$ is indeed promising and could serve as a critical tool to further examine the characteristics of the fetal genes and the underlying cellular and molecular processes (Figure 1). However, several key points remain that warrant further investigation: (1) Is there a specific phenotype of the pre-existing cardiomyocytes that proliferates and migrates, and the population of the specific phenotype is lost by the end of the first week of life? (2) Is there a specific population of neonatal cardiomyocytes that undergo cell-cycle arrest? If this is true, what are the underlying mechanisms? (3) What is the effect of the myocardial wall thickness, orientation of cardiomyocytes, regeneration of epicardium and endocardium, and normal size and density of coronary vessels on heart regeneration? (4) What are the genetic and epigenetic mechanisms, the characteristics of the fetal genes that are re-expressed in neonatal cardiomyocytes to control cell proliferation, and paracrine effects after injury to promote regeneration? (5) Is the neonatal heart regeneration dependent on the duration of coronary occlusion, type of injury, and the infarct size? and (6) How could we reprogram adult cardiomyocytes to differentiate into a proliferative phenotype? Could this be achieved by simulating the hypoxic fetal environment to upregulate the genes and other endogenous regulators critical for heart regeneration? Is there a possibility to express key regenerative genes in the myocardium using cellular reprogramming strategies or bioengineered gel? ${ }^{10,11}$
The findings from such studies will advance our knowledge using clinically relevant models of neonatal heart regeneration following ischemic injury and will be critical in identifying innate mechanisms and molecular targets to develop clinically feasible strategies in the regeneration of adult heart. Certainly, the concept is tantalizing, but in reality this remains a distant goal.

\section{References}

1. Tan CMJ, Lewandowski AJ. The transitional heart: from early embryonic and fetal development to neonatal life. Fetal Diagn Ther. 2020;47:373-86.

2. Brown SM, Larsen NK, Thankam FG, Agrawal DK. Fetal cardiomyocyte phenotype, ketone body metabolism, and mitochondrial dysfunction in the pathology of atrial fibrillation. Mol Cell Biochem. 2021;476:1165-78.

3. Porrello ER, Mahmoud AI, Simpson E, Hill JA, Richardson JA, Olson EN, et al. Transient regenerative potential of the neonatal mouse heart. Science. 2011;331: 1078-80.

4. Zhu W, Zhang E, Zhao M, Chong Z, Fan C, Tang Y, et al. Regenerative potential of neonatal porcine hearts. Circulation. 2018;138:2809-16.

5. Lam NY, Sadek HA. Neonatal heart regeneration. Circulation. 2018;138:412-23.

6. Sadek HA, Porrello ER. Neonatal heart regeneration: moving from phenomenology to regenerative medicine. J Thorac Cardiovasc Surg. 2020;159:2451-5.

7. Wang H, Paulsen MJ, Hironaka CE, Shin HS, Farry JM, Thakore AD, et al. Natural heart regeneration in a neonatal rat myocardial infarction model. Cells. 2020; 9:229.

8. Haubner BJ, Schneider J, Schweigmann U, Schuetz T, Dichtl W, VelikSalchner C, et al. Functional recovery of a human neonatal heart after severe myocardial infarction. Circ Res. 2016;118:216-21.

9. Wang H, Hironaka CE, Mullis DM, Lucian HJ, Shin HS, Tran NA, et al. A neonatal leporine model of age-dependent natural heart regeneration after myocardial infarction. J Thorac Cardiovasc Surg. 2022;164:e389-405.

10. Agrawal DK, Thankam FG. Commentary: divine decree or a novel panacea in CRISPR-Cas9-steered cellular reprogramming in the fate of failing heart. $J$ Thorac Cardiovasc Surg. May 24, 2020 [Epub ahead of print].

11. Agrawal DK, Siddique A. Commentary: rejuvenation of "broken heart" with bioengineered gel. J Thorac Cardiovasc Surg. 2019;157:1491-3. 